\title{
Research on Design and Implementation of Personal Website
}

\author{
Rongfu Wang ${ }^{1}$, Ying Peng ${ }^{2}$ \\ ${ }^{12}$ Nanchang Institute of Science \& Technology, Nanchang, Jiangxi, China, 330108
}

KEYWORDS: Design, Implementation, Personal Website

\begin{abstract}
In recent years, with the rapid development of network technology, computer network has gradually penetrated into every corner of life, personal journal published by web browsing and content of interest to a growing concern in today's society has gradually become self-display and a new way to discover talent, personal websites came into being. This paper studies the design and implementation of individual sites.
\end{abstract}

\section{Introduction}

Steady improvement at this stage, with the rapid development of the Internet and penetration, people have to live with the integration of the Internet, the Internet has become an important form of interpersonal exchanges between people and society. The personal website, happens to be one of the inevitable product of the process of rapid development of the Internet world, people through personal website design and development, not only personal preferences, a service or product, and other related information, penetrate into the Internet, you can also use personal website set up personal online presence, build up a personal recommendation, to show themselves, interact network platform, to shorten the distance between people, between people and society.

Website focus reflects a personal preference of interest, and can be divided into sections according to their custom-defined style. This article is designed personal Web site using the popular programming technology ASP (Active Server Pages) implementation, the database with ACCESS. In this paper, the demand for systems analysis and design, database design, programming and implementation of key technologies and system test cases into detail.

General idea of design of the site is in a person's management, users browse through the author's personal website to communicate with the author, and can get some of the latest news or information on a personal Web site, and can comment on articles of interest, can the author has set a good link to access online message to the author. Administrators all through the background to manage the entire site, do not need to view the source files or code, which functions include add articles, edit or delete the article to view the message, filtered reviews words, change the administrator nickname, and so on.

\section{Related Research on Website}

Personal website design and development process, the site needs to do analysis to determine the development languages and database systems website. This system is mainly based on personal website PHP development environment, through PHP and MySQL database technology combined in highly interactive Internet platform, based on the framework to achieve functional personal website, allowing users in the browser easy and fast visit the website system, browse the relevant information they need, so that not only save and manage documents provide a great convenience, but also greatly enhance interactive web pages to enhance the user experience of the site system. 
Personal website is article log, album pictures, personal information, message boards, and many other functions in one network platform. Website individual as the center, set up a very own customized personalized network platform, highlighting their individual needs, ease of use, features to help individuals use the Internet to conduct a comprehensive display of the information, in order to achieve various forms of information Get organized, think, express, share and exchange purposes. By User comments on the article, a message on the message board, listen to the voices from the Internet and communicate with others to achieve their interactive effects.

Making dynamic web site creation and system design and the development, PHP development has become the mainstream use today. Because PHP is a versatile open source scripting language that has absorbed the characteristics of a variety of development languages, easy to use, simple operation, it won a lot of web design and development of all ages. PHP integrated in HTML and scripting language and its simple working interface, the entry threshold is relatively low, the code is simple, fast, high efficiency, wide use, supports almost all popular databases and operating systems, PHP is a powerful feature enables personal website with better network platform interoperability and interactivity.

\section{The Goals of Personal Website Design}

With reference to various forms of personal sites, combined with the current status of the network, a more ideal personal information management Web site with the following features:

(1) within the web site allows anyone to view the information; (2) allows anyone to view information classified by section; (3) allows anyone by article type, title authors to query data; (4) allows you to browse Announce; (5) user logon; (6) See own published data; (7) in the address book to add information; (8) Log status.

\section{The Development Tools of Website}

Dreamweaver is developed by the United States MACROMEDIA set web site creation and management of web editor-in-one, it is a visual web development tools for professional web designers first set of special development, which allows you to easily create cross-platform web restrictions.

Photoshop is recognized as the best general the graphic design software. Its user interface is straightforward, functional, stable performance, therefore, in almost all of the advertising, publishing, software companies, Photoshop is the tool of choice for the plane.

Flash is an American MACROMEDIA company in June 1999 launched the excellent web page animation software. It is an interactive animation design tool. It can be used together music, sound, animation, and rich innovative interface to create high quality web dynamic effects.

\section{Feasibility Analysis}

Feasibility analysis system includes economic viability, technical feasibility on the feasibility of the operation.

And benefit from the development of the system in terms of the relationship, development of the system does not need to spend too much money. The system uses asp that can develop interactive, high-performance Web database application technology development; development of the system as quickly as possible to release information for the user to reduce the notification time information; more timely understanding of the information unit of the publishing situation. Therefore, from a financial feasibility is concerned, it is feasible. 
According to the existing conditions of technical equipment and preparing enrichment technology and equipment, systems implementation is technically feasible. In terms of equipment, computer memory, external memory capacity, etc. can be input and output devices to meet the needs on the basis of the original. From the entire system structure, it can be achieved on the basis of the existing local area network servers, workstations and network components on. In addition, the system's developers have computer knowledge, there are certain programming foundation, known and master the knowledge and skills databases and networks, can develop web-based information management system for foreign projects.

The system in operation is very simple. For users, only need to have a basic Internet technology on the line. Thus, the system is feasible in the operation.

\section{Development and Design of Personal Websites}

For website design and development, we must first determine the theme of the site. Select the appropriate theme of the site, so that small and fine positioning. If the theme of the site location process, not accurate enough, blind design, arbitrary, then the site will allow users to feel the theme is not obvious, it not only causes a waste of the developer's work, but also the loss of the site highlights the promotion of the site in the future and operational processes, causes difficulties. Therefore, to accurately grasp the theme of the site throughout the website design and development process is very important, and the future development of the site is closely related. Personal website showcases information individuals want to advertise to personal interests as the center, diaries and essays about the photo album to display the contents of their personal lives, emotions and work.

Image of the site occupies a very important position in website design and development process. Will affect the image of the site visitors first impression of the site, the site in a different color scheme will produce different results, different results will form different visual impact, thus affecting the website visitors emotions. In the website design process will involve site with many elements, such as the site logo, site colors, fonts and images site elements, different elements combined together. Meanwhile, the various elements of the site layout module classification will affect the overall quality of the site. A well-designed Web site, its content is largely considered the habit of most users use the site, allowing users to sites with a better combination. Good website can make website content distinct image, highlighting the theme of the site, allowing users to achieve convenient operation, clear results, clearly feel the unique characteristics of the site, the site for the promotion and operations hit a solid foundation.

Effects and characteristics of the site is determined by the function of the site itself, the page layout of the site's impact on the end-user experience, therefore, the page layout of the site has played a crucial role in the website system development process. In the page layout process, we should follow the following points:

Determine the width of the site pages. When the page structure is positioned, there is a very important parameter is determined that the width of the page. Determine the page width is usually fixed-pixel display mode and adaptive mode. With the change in user habits change and public use of the display size, the width of the web page should follow the most popular, the most popular page size.

Personal website system in the head and the bottom of the layout to the page separation mode, the head and the bottom of the page layout and control are unified through the head and the bottom of the document file, by way of calling different pages to achieve precise layout of the page and control. 
Design the main content of the page layout. Most sites use the traditional table-based layout displays pages of information, although it is easy to create a form, but the form will generate a lot of difficult to read and maintain code in Web browsers actual process there are some compatibility issues. So this website in the main content page layout using DIV layer with a combination of Cascading Style appearances layout, flexible control of web design, site content separated structure, performance and behavior. Express the contents to be clear and concise, allowing users to browse at a glance during the visit.

The personal website database system use MySQL database. MySQL in the WEB application is the best RDBMS: one (Relational Database Management System relational database management system) applications. MySQL relational database data can be saved in separate tables rather than putting all the data in a large warehouse, not only fast access speed, flexibility is also very strong. SQL language used by MySQL is the most common standardized language used to access databases. MySQL software uses a dual licensing policy, it is divided into Community Edition and the commercial version, due to its small size, high speed, low cost of ownership, especially open source this feature, the development of small and medium websites have chosen MySQL as the database website.

With the rapid development of broadband wireless access technology and mobile terminal technology, people are eager to be able to anytime, anywhere and even be able to easily access information and services from the Internet during the move, and the rapid development of mobile Internet emerged. Personal website system development process, but also the deployment of the mobile Internet in each node, the mobile version of the website system optimization, improve, in order to enhance the user experience, bundled with more users, operators lay the foundation for the promotion of personal site later.

\section{Conclusion}

In short, personal website system design and development process, to achieve the functionality of the site at the same time, always with the user experience as the center, designed to enhance interactive website. Only allow authorized users, in line with the requirements of users, the latter operation of the site in order to have better development, personal websites to achieve its unique value.

\section{REFERENCE:}

[1] Guo Minghua, Guoming Lan, Li Qing. ASP technology to access the database. Computer Learning, 2005, (03).

[2] Hu Xiaorui. Flood Control System Design and Implementation based AsP. Computer Engineering, 2005, (20).

[3] Liu Haihong, Qi Yinan, Liu Boying. ASP pages use database import text data to achieve high-performance computing technology,2000. (05).

[4] Liu Haihong, Qi Yinan, Liu Boying. Implement a database with ASP pages to import text data. Industrial control computer, 2001,(01).

[5] Liu Wenying. ASP Application negligent database. China Science and Technology Information, 2004, (03). 